\title{
Role of CT perfusion imaging in evaluating the effects of multiple burr hole surgery on adult ischemic Moyamoya disease
}

\author{
Dong Wei Dai • Wen Yuan Zhao • Yong Wei Zhang • \\ Zhi Gang Yang • Qiang Li • Bing Xu $\cdot$ Xiao Long Ma • \\ Bing Tian • Jian Min Liu
}

Received: 27 May 2013 / Accepted: 9 October 2013 /Published online: 24 October 2013

(C) The Author(s) 2013. This article is published with open access at Springerlink.com

\begin{abstract}
Introduction To evaluate the effects of the multiple burr hole (MBH) revascularization on ischemic type adult Moyamoya disease (MMD) by computed tomography perfusion (CTP). Methods Eighty-six ischemic MMD patients received CTP 1 week before and 3 weeks after MBH operation. Fifty-seven patients received it again at 6 month and underwent digital subtraction angiography (DSA) and mRS follow-up. Cerebral blood flow (CBF), cerebral blood volume (CBV), mean transit time (MTT), time to peak (TTP), and relative values of ischemic symptomatic hemispheres were measured. Differences in preand post-surgery perfusion $\mathrm{CT}$ values were assessed.

Results There were significant differences of CBF, TTP, and relative time to peak (rTTP) in ischemic hemisphere between 1 week before and 3 weeks after surgery, and no significant difference in relative cerebral blood flow (rCBF), CBV, relative cerebral blood volume (rCBV), MTT, relative mean transit time (rMTT). According to whether there was
\end{abstract}

Dong Wei Dai and Wen Yuan Zhao contributed equally to this paper.

Electronic supplementary material The online version of this article (doi:10.1007/s00234-013-1291-1) contains supplementary material, which is available to authorized users.

D. W. Dai · W. Y. Zhao · Z. G. Yang • Q. Li · J. M. Liu $(\bowtie)$ Department of Neurosurgery, Changhai Hospital, Second Military Medical University, No. 168 Changhai Road, Yangpu District, Shanghai 200433, People's Republic of China

e-mail: chstroke@126.com

Y. W. Zhang

Department of Neurology, Changhai Hospital, Second Military

Medical University, Shanghai, People's Republic of China

B. Xu $\cdot$ X. L. Ma $\cdot$ B. Tian

Department of Radiology, Changhai Hospital, Second Military

Medical University, Shanghai, People's Republic of China symptom improvement or not on 3 weeks after $\mathrm{MBH}$, the rTTP value was not statistically significant in the patients whose symptoms were not improved at all on 3 weeks after operation. Six-month follow-up showed that $\mathrm{CBF}, \mathrm{rCBF}$, and rCBV values were significantly higher than those before operation. Postoperative MTT, TTP, rMTT, and rTTP values were significantly lower than those before operation.

Conclusion CTP is a sensitive method to obtain functional imaging of cerebral microcirculation, which can be a noninvasive assessment of the abnormalities of intracranial arteries and cerebral perfusion changes in MMD before and after surgery. CBF and TTP map, especially the relative values of TTP, seems to have the capability of being quite sensitive to the presence of altered brain perfusion at early time after indirect revascularization.

Keywords Moyamoya disease $\cdot \mathrm{CT}$ perfusion $\cdot$ Multiple burr hole surgery $\cdot$ Ischemic

$\begin{array}{ll}\text { Abbreviations } \\ \text { MMD } & \text { Moyamoya disease } \\ \text { MBH } & \text { Multiple burr hole } \\ \text { EDAS } & \text { Encephaloduroarteriosynangiosis } \\ \text { EMS } & \text { Encephalomyosynangiosis } \\ \text { EDAMS } & \text { Encephaloduroarteriomyosynangiosis } \\ \text { CTP } & \text { Computed tomography perfusion } \\ \text { CTA } & \text { Computed tomography angiography } \\ \text { Xe-CT } & \text { Xenon-enhanced computed tomography } \\ \text { SPECT } & \text { Single photon emission computed tomography } \\ \text { PET } & \text { Positron emission tomography } \\ \text { DSA } & \text { Digital subtraction angiography } \\ \text { MRI } & \text { Magnetic resonance imaging } \\ \text { TIA } & \text { Transient ischemic attack } \\ \text { ROI } & \text { Regions of interest } \\ \text { STA } & \text { Superficial temporal artery }\end{array}$




$\begin{array}{ll}\text { mRS } & \text { Modified Rankin scale } \\ \text { AIF } & \text { Arterial input function } \\ \text { MTT } & \text { Mean transit time } \\ \text { CBV } & \text { Cerebral blood volume } \\ \text { CBF } & \text { Cerebral blood flow } \\ \text { TTP } & \text { Time to peak } \\ \text { ECA } & \text { External carotid artery } \\ \text { ACA } & \text { Anterior cerebral artery } \\ \text { MCA } & \text { Middle cerebral artery } \\ \text { PCA } & \text { Posterior cerebral artery }\end{array}$

\section{Introduction}

Moyamoya disease (MMD), characterized by progressive stenosis or final occlusion of the internal carotid artery and the circle of Willis, is an idiopathic occlusive cerebrovascular disease of unknown etiology. Its common clinical manifestations were intracranial hemorrhage in adults and cerebral ischemia in children and adolescents [1-3]. According to our knowledge, no treatment can reverse the process of primary MMD. Revascularization surgery proves to be effective in preventing stroke in patients with Moyamoya [4]. Surgical procedures for indirect bypass are specific for MMD. There are various methods for indirect bypass, including multiple burr hole $(\mathrm{MBH})$ surgery, encephaloduroarteriosynangiosis (EDAS), encephalomyosynangiosis (EMS) and encephaloduroarteriomyosynangiosis (EDAMS), etc. Indirect bypass surgery that induces spontaneous angiogenesis between the brain surface and the vascularized donor tissues is technically simple and has been widely used.

Many techniques such as digital angiography, transcranial Doppler, computed tomography perfusion (CTP), computed tomography angiography (CTA), xenon-enhanced CT (Xe$\mathrm{CT}$ ), positron emission tomography (PET), and single photon emission computed tomography (SPECT) with acetazolamide challenge have all been used in the evaluation of patients with MMD. CTP, a tool to obtain functional imaging of cerebral blood flow, can efficiently identify abnormal brain perfusion other than conventional CT and MRI [5]. In addition, CTP can be performed quickly and provide valuable data for diagnosis. In this study, our purpose was to evaluate the value of the application of CTP in investigating cerebral hemodynamic changes in ischemic type MMD before and after surgical revascularization of $\mathrm{MBH}$.

\section{Material and methods}

Patients

From Mar. 2009 to Feb. 2013, a total of 86 patients with ischemic type definite MMD diagnosed by digital subtraction angiography (DSA) in Changhai Hospital, were included in this study. Of the 86 patients (age range, 19.0-68.0 years; mean age, 37.63 years), there were 31 men and 55 women. This study was approved by the local ethical committee (Institutional Review Board of Changhai Hospital, Second Military Medical University), and written informed consents were obtained. The diagnostic criteria for MMD were based on the guidelines published in 1997 by the Research Committee on the Spontaneous Occlusion of the Circle of Willis of the Ministry of Health and Welfare, Japan [2]. Patients with unilateral lesions (probable MMD and Moyamoya syndrome) were excluded from our study; subjects were not considered with particular potential risk factors for MMD, such as irradiation to the head or neck, autoimmune disease, atherosclerosis, meningitis, brain neoplasm, Down's syndrome, neurofibromatosis, and sickle cell disease. All the $86 \mathrm{MMD}$ patients were ischemic type definite MMD individuals. Hemorrhagic definite MMD patients were also excluded from our study. Clinical manifestations were all ischemic symptoms such as transient ischemic attack (TIA), dizziness, headache, limb weakness, sudden syncope and visual field defect, etc. Suzuki classification of 86 regions of interest (ROI) sides was: Suzuki stage I, $15.1 \%$ (13/86); II, $32.5 \%$ (28/86); III, $45.3 \%$ (39/86); IV, $5.8 \%(5 / 86)$; and V, $1.2 \%(1 / 86)$. The diagnostic criteria of Suzuki stage was based on the guidelines [2].

All the 86 definite MMD patients underwent MBH surgeries on bilateral sides. On the one hand, burr hole surgery could improve the symptomatic hemisphere; on the other hand, it could also prevent further stroke events on nonsymptomatic hemisphere during the natural progress of MMD. The detailed procedures of burr hole surgery were essentially the same as those for burr hole openings for external ventricular drainage. The operation was performed under local anesthesia. The superficial temporal artery (STA) was located by Doppler ultrasonography to avoid sacrificing it at the time of skin incision. There were 14-16 holes for each patient. After trephination, the dura and arachnoid were opened widely preserving the meningeal arteries under microscope.

Every individual received CTP examination 1 week before operation and CTP reexamination 3 weeks after $\mathrm{MBH}$ operation. Fifty-seven patients received it again at 6-month follow-up. All patients were graded by the modified Rankin scale (mRS) at admission and discharge from hospital. Fiftyseven patients underwent DSA examination and $\mathrm{mRS}$ followup at 6 months after MBH surgery. Symptom improvement was defined as follows: decrease or even disappearance of the TIA and sudden syncope frequency; dizziness or headache relief, or even disappearance; improved limb weakness or visual field defect. 
CT scanning and image processing

CT scan was performed in the transverse plane using a 64slice multi-detector CT scanner (SOMATOM AS 128; Erlangen, Germany). CTP consisted of a 60-s series, with 30 gantry rotations performed in cine mode during the intravenous administration of iodinated contrast material. Images were acquired and reconstructed at a temporal sampling rate of 1 image per $2 \mathrm{~s}$, resulting in a series of 20 images for each assessed section. After unenhanced CT scan of the whole brain, eight adjacent, 8-mm-thick sections were selected by starting at the level of the basal ganglia. A 50-ml bolus of nonionic contrast media (Ultravist 370, Schering Health Care Ltd., Burgess Hill, UK) was administered into an antecubital vein by using a power injector (Stellant Injection System; Indianola, PA, USA) with an injection rate of $5 \mathrm{ml} / \mathrm{s}$. We used volume shuttle scanning mode, which could get $80 \mathrm{~mm}$ cerebral perfusion image in one time, to display range from basal ganglia region to brainstem region. At the same time, a CTA image could also be obtained.

\section{Post-processing procedure}

The brain plain images, dynamic CTA, and brain perfusion images were reconstructed from source data acquired with dynamic scans. The dynamic CT angiographic images were generated from volumetric data acquired in the arterial, capillary, and venous phases of CTP source data. The volume-rendered images of cerebral arteries performed at the peak of the arterial time-attenuation curve were the same as conventional CTA images using advanced vessel analysis software installed in the workstation. CTP data were analyzed using Siemens perfusion software (Siemens Medical Systems), which relied on the central volume principle to calculate perfusion parameters from the time-concentration curve. The software applies curve fitting by a least-mean squares method to obtain mathematical descriptions of the time-attenuation curves, and the mean transit time (MTT) map was calculated by a closed-form (noniterative) deconvolution operation from the time-concentration curve of a particular voxel and the arterial input function. The first artery to reach peak enhancement on the time-attenuation curve was selected. The vein with the largest area under the time-attenuation curve was selected as the venous outflow function. For each voxel, the cerebral blood volume (CBV) map was calculated from the areas under the timeconcentration curves. The cerebral blood flow (CBF) map for each voxel was finally calculated according to the following equation combining the $\mathrm{CBV}$ and MTT values: $\mathrm{CBF}=\mathrm{CBV} / \mathrm{MTT}$. Large cortical vessels were automatically excluded via brain perfusion software. Two experienced neuroradiologists independently drew two standardized elliptical mirrored ROIs manually on the above basal ganglia section level of the reference CT image over the cortical gray matter of ACA or MCA territory (ROIs were placed on cortical regions in the anterior cerebral artery territory shown in Fig. 1). Perfusion CT absolute values of one hemisphere in the region of $\mathrm{MCA}$ distribution and contralateral mirroring areas in functional maps were measured; from each ROI, CBF, CBV, MTT, and time to peak (TTP) were calculated. The relative CTP values in our study were defined as the ratios between absolute CTP values of symptomatic side and absolute CTP values of pons region. There were relative cerebral blood flow ( $\mathrm{rCBF})$, relative cerebral blood volume (rCBV), relative time to peak (rTTP), and relative mean transit time (rMTT). Symptomatic sides were decided by two experienced neurosurgeons independently from clinical manifestations and CT and MRI images.

\section{Dyna CT processing}

After DSA, the dual volume visualization technique was employed to check the relationship between vessel and skull (burr hole). Firstly, a mask and a fill rotational scanning were performed by angiography system. Then, reconstructed mask volume and subtracted fill volume were loaded in a dedicated dual-volume mode, i.e., a bone preset for the mask volume and a vessel preset for the fill volume. In the end, both skull and vessel were visualized at the same time in the volume rendering technique mode.

\section{Statistical analysis}

For CTP parameters, the mean of ROI values on symptomatic hemisphere was calculated before and after $\mathrm{MBH}$ surgery. Data were expressed as mean \pm SD. Values with $P<0.05$ were considered statistically significant. All raw data were analyzed using statistical software SPSS 13.0 Statistical Software Package (SPSS Inc., Chicago, IL).

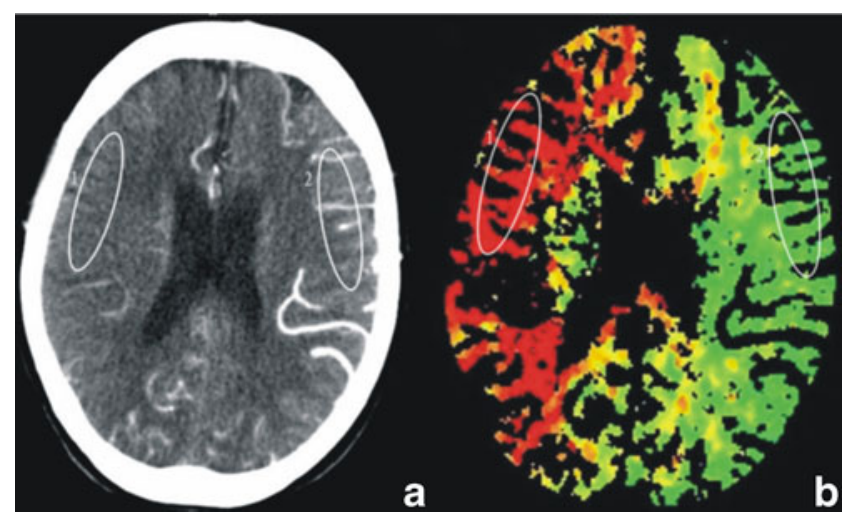

Fig. 1 ROIs were placed on cortical regions in the anterior cerebral artery territory ROIs were drawn in a the reference CT image and b TTP map 


\section{Results}

\section{CT scanning and CT angiography}

Abnormal densities in brain tissue were detected in most cases, among which 79 cases (92\%) had brain infarction including 17 cases (19\%) of encephalomalacia and 10 cases (12\%) of atrophy of brain. Normal density was seen in seven cases $(8 \%)$. The severe stenosis and occlusion of multiple cerebral vessels could be well demonstrated on volumetric CTA, and increased quantity of the external carotid arterial system and new collateral artery in burr hole areas could be clearly seen in the CTA post-MBH surgery.

\section{DSA/dyna CT and mRS}

All patients underwent DSA before operation, and were diagnosed with definite MMD. Fifty-seven patients received DSA at 6-month follow-up completely. Of the 57 patients, 42 individuals $(73.68 \%$ ) had great new collaterals from external carotid artery (ECA) system to cortical region in hole areas of ischemic symptomatic hemispheres. Dyna CT reconstruction demonstrated that more than $50 \%$ holes showed collaterals in these patients (typical follow-up case was shown in Fig. 2). Fourteen patients $(24.56 \%)$ had rare newly built collaterals in hole areas, and only one patient $(1.76 \%$ ) had no collateral compared to preoperational situation. Additional mRS followups were also performed with the 57 patients who underwent DSA at 6 months after MBH surgery. Compared with mRS performed at admission before operation of the same individuals, 49 patients had a decreased mRS (85.9\%) and the symptoms before operation in the 49 individuals were all improved. The follow-up results revealed good clinical outcomes from the effect of $\mathrm{MBH}$ indirect revascularization.
CT perfusion

Our study focused on the perfusion change only on the symptomatic hemisphere, so we selected the ROIs of the symptomatic side for comparison and statistical analysis.

Based on the pre- and postoperative CTP parameters, Table 1 displayed the comparisons of CBF, CBV, TTP, MTT, and relative values between preoperative and postoperative 3 weeks after surgery. There were significant differences of CBF, TTP, and rTTP $(P<0.05)$ in ischemic symptomatic hemisphere between 1 week before and 3 weeks after surgery, and no significant difference in $\mathrm{rCBF}, \mathrm{CBV}$, rCBV, MTT, and rMTT ( $P>0.05$ for all) were found. Typical $\mathrm{CT}$ perfusion changes pre- and 3 weeks post-MBH surgery were shown in Fig. 3.

Further assessment of normalized data was demonstrated in Table 2. All patients were divided into two groups according to symptom improvement on 3 weeks after $\mathrm{MBH}$ surgery. Thirty-two cases (37\%) showed symptom improvements, and 54 cases $(63 \%)$ displayed none. There were both significant differences in TTP values $(P<0.05)$ in the two groups; however, the differences in rTTP were detected: rTTP in the symptom-improved group was statistically significant $(P<$ $0.05)$, but the rTTP was not statistically significant $(P>0.05)$ in the patients whose symptoms were not improved at all on 3 weeks after operation. Other normalized data (rCBF, rCBV, rMTT) were not statistically significant $(P>0.05)$ no matter whether the symptoms improved or not.

Six-month follow-up data (shown in Table 3) from the 57 patients revealed that the postoperative $\mathrm{CBF}, \mathrm{rCBF}$, and $\mathrm{rCBV}$ values in the region of MCA were significantly higher than those before operation ( $P<0.05$ for all). Postoperative MTT, rMTT, TTP, and rTTP values were significantly lower than those before operation $(P<0.05$ for all).

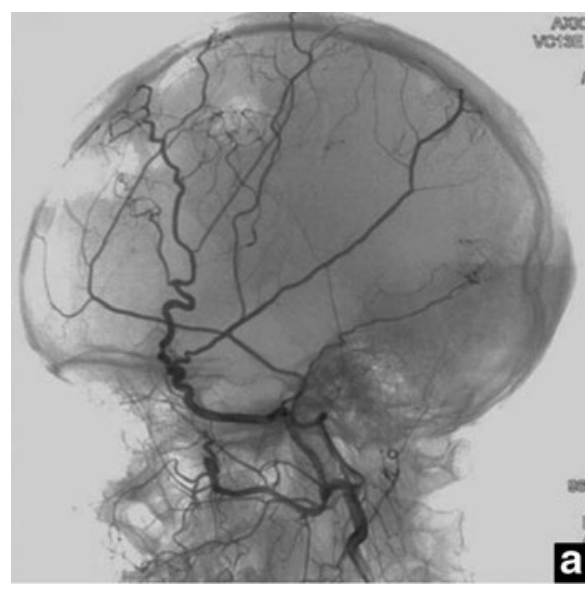

Fig. 2 DSA and Dyna-CT reconstructive imaging follow-up (6 months) after $\mathrm{MBH}$ surgery. Multiple burr holes in frontal regions are demonstrated in early phase of right external carotid angiograms without

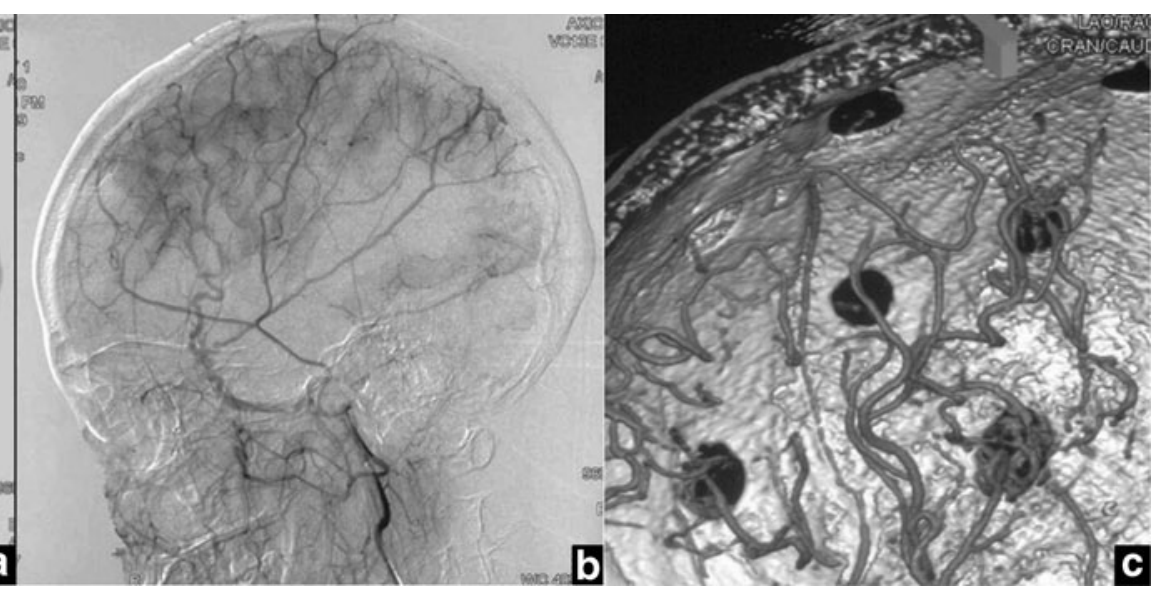

digital subtraction (a). Neovascularization is rich through the burr holes (b). Dyna CT reconstructive image (c) after DSA shows great new collaterals from ECA system to cortical region in hole areas 
Table 1 Comparison of CT perfusion parameters between preoperative and postoperative 3 weeks after $\mathrm{MBH}$ indirect revascularization

\begin{tabular}{llll}
\hline & Preoperative & $\begin{array}{l}\text { Postoperative } \\
3 \text { weeks }\end{array}$ & $p$ Value \\
\hline $\mathrm{CBF}\left(\mathrm{ml} \cdot 100 \mathrm{~g}^{-1} \cdot \mathrm{min}^{-1}\right)$ & $54.65 \pm 7.78$ & $56.88 \pm 6.12$ & $0.013^{*}$ \\
$\mathrm{rCBF}$ & $0.68 \pm 0.44$ & $0.69 \pm 0.87$ & 0.076 \\
$\mathrm{CBV}\left(\mathrm{ml} \cdot 100 \mathrm{~g}^{-1}\right)$ & $5.68 \pm 2.45$ & $5.85 \pm 2.86$ & 0.574 \\
$\mathrm{rCBV}$ & $0.76 \pm 0.07$ & $0.78 \pm 0.04$ & 0.458 \\
$\mathrm{MTT}(\mathrm{s})$ & $6.42 \pm 0.91$ & $6.39 \pm 0.82$ & 0.145 \\
$\mathrm{rMTT}$ & $1.67 \pm 0.63$ & $1.71 \pm 0.21$ & 0.214 \\
$\mathrm{TTP}(\mathrm{s})$ & $20.62 \pm 0.91$ & $17.46 \pm 0.53$ & $0.000^{*}$ \\
$\mathrm{rTTP}$ & $1.82 \pm 0.24$ & $1.51 \pm 0.03$ & $0.042^{*}$ \\
\hline
\end{tabular}

The data are presented as mean $\pm \mathrm{SD} ; n=86$

$* P<0.05$ (statistically significant)

\section{Discussion}

MMD is an uncommon cerebrovascular disorder that is characterized by progressive occlusion of the supraclinoid internal carotid artery and its main branches within the circle of Willis. The natural history of MMD is variable, whose progression can be slow or fulminant, with rare intermittent events or with rapid neurologic declines [6]. MMD, featured by recurrent episodes of cerebral ischemia or intracranial hemorrhage, is closely related with hemodynamic changes. Patients with MMD have reduced CBF and increased CBV, MTT, and TTP. These hemodynamic changes are explained by decreases in the cerebral perfusion pressure and associated vasodilation [7]. Due to the decline in reserve capacity of cerebral circulation, acetazolamide stress test still cannot detect the normal brain blood flow response. An understanding of cerebral hemodynamics is thought to be essential in determining the indicators for vascular reconstruction in patients with MMD [8]. In order to identify patients who suffer from hemodynamic cerebral insufficiency and enable them to benefit from cerebral revascularization procedures, doctors use CT scanning to reliably and accurately measure the critical cerebrovascular reserve capacity [9]. CT perfusion was used to evaluate the cerebral blood flow in this study. In 1991, Miles et al. [10] developed color perfusion imaging technique with the combination of the fast CT scanning and computer image processing system, and proposed the conception of "CT perfusion." Since then, this technology has been developing rapidly and widely used in clinical applications. The assessment of cerebral ischemia by means of perfusion parameters derived from perfusion $\mathrm{CT}$ provides valuable information to predict outcomes. CBF refers to the blood supply to the brain in a given time. CBV is the vascular bed volume of capillaries and large blood vessels in ROI; MTT refers to the average time that contrast agent spends in passing by ROI; TTP is the time interval from the contrast agent's first arrival of the artery in scanned layers to peak bolus of contrast agents in the brain. CBF, CBV, TTP, and MTT images are interpreted together on a workstation that permits the use of visual assessment which is combined with quantitative analysis with ROI. Regions of prolonged TTP and MTT often represent regions of decreased perfusion.

Many techniques have been used in the evaluation, for example, conventional digital subtractive angiography, transcranial Doppler, MRI, CTA, CTP, Xe-CT, PET, and SPECT with acetazolamide challenge, etc. However, each technique has its own merits and drawbacks. SPECT has been regarded as a reference standard, in terms of evaluating hemodynamic status in MMD [11]. However, due to tracer kinetics, SPECT usually has to be performed in a 2-day setting. What is more, it provides less morphologic information than CT or MRI. As for MRI perfusion, the quantification of $\mathrm{CBF}$ is more complex than with CTP
Fig. 3 CTP image changes preand 3-week post-MBH surgery. Images show cerebral

hemodynamic changes pre- and 3 -week post-MBH surgery. Axial perfusion $\mathrm{CT}$ images show reduced a $\mathrm{CBF}$, increased $\mathbf{b} \mathrm{CBV}$, delayed $\mathbf{c}$ MTT, and $\mathbf{d}$ TTP in the region of right hemisphere. The 3week postoperative perfusion $\mathrm{CT}$ images show slightly increased $\mathbf{e}$ $\mathrm{CBF}$, almost no change in $\mathbf{f} \mathrm{CBV}$, slightly reduced $\mathbf{g}$ MTT, and significantly reduced $\mathbf{h}$ TTP in the right hemisphere

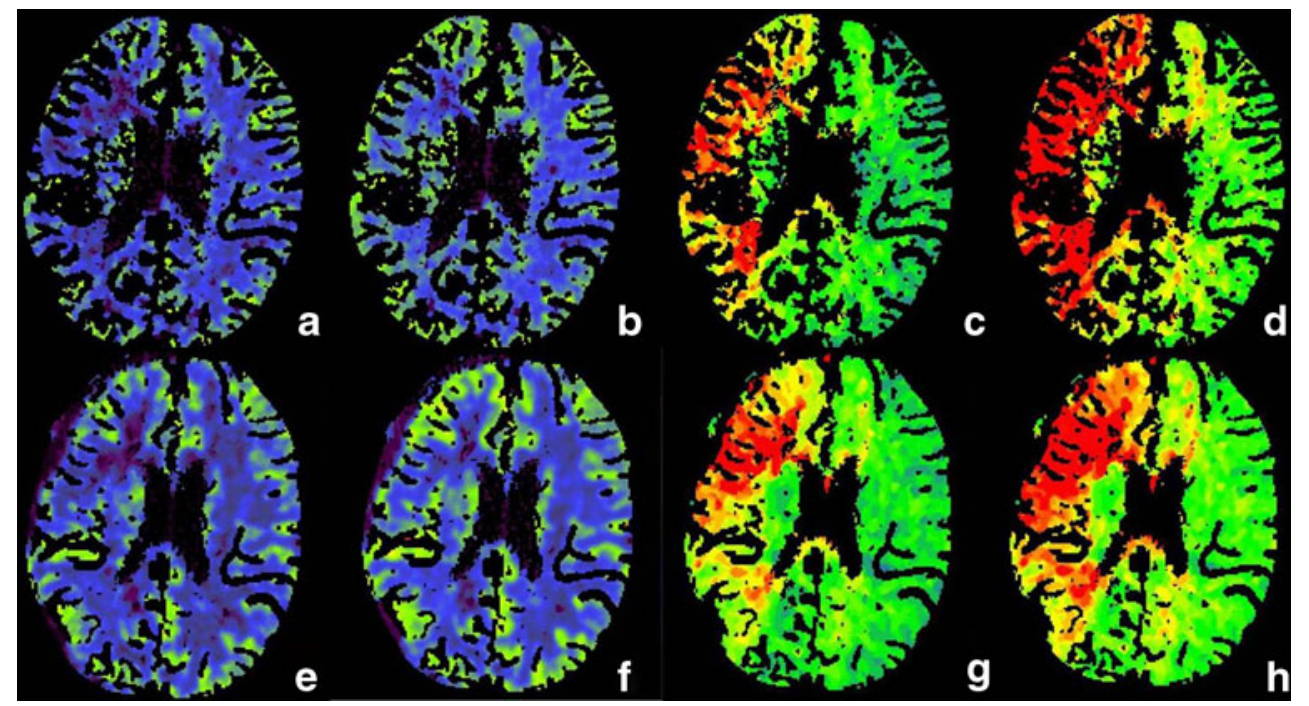


Table 2 Normalized data of CTP between 1 week before and 3 weeks after MBH indirect revascularization (symptom improved or not)

\begin{tabular}{|c|c|c|c|c|c|c|c|c|}
\hline & \multicolumn{4}{|c|}{ Symptom improved (32 cases) } & \multicolumn{4}{|c|}{ Symptom not improved (54 cases) } \\
\hline & $\mathrm{rCBF}$ & $\mathrm{rCBV}$ & rMTT & rTTP & $\mathrm{rCBF}$ & $\mathrm{rCBV}$ & rMTT & rTTP \\
\hline Pre & $0.69 \pm 0.22$ & $0.77 \pm 0.38$ & $1.67 \pm 0.22$ & $1.84 \pm 0.28$ & $0.66 \pm 0.48$ & $0.76 \pm 0.02$ & $1.66 \pm 0.18$ & $1.79 \pm 0.32$ \\
\hline Post & $0.70 \pm 0.59$ & $0.77 \pm 0.67$ & $1.72 \pm 0.54$ & $1.32 \pm 0.37$ & $1.67 \pm 0.33$ & $0.78 \pm 0.39$ & $1.70 \pm 0.32$ & $1.65 \pm 0.13$ \\
\hline$P$ & 0.064 & 0.548 & 0.163 & $0.008 *$ & 0.130 & 0.132 & 0.224 & 0.134 \\
\hline
\end{tabular}

The data are presented as mean $\pm \mathrm{SD}$; Values are considered statistically significant when $P<0.05$.

because the relationship between the signal intensity and the gadolinium concentration is not always linear [3]. Furthermore, the Xe-CT can be used for accurate quantitative evaluation of regional cerebral blood flow and has a wider clinical application.

CT perfusion study has some disadvantages. First of all, it is likely to have a high radiation dose, and it is also quite hard to accurately draw MCA territories before and after $\mathrm{MBH}$ revascularization surgery. If both sides are of the similar decline in cerebral perfusion at preoperative evaluation of hemodynamics in MMD patients with bilateral damage, it may underestimate the damage to cerebral blood flow. Another limitation concerns the measurement of the true arterial input function (AIF). AIF is calculated from major cerebral vessels; however, in MMD patients, anterior cerebral artery (ACA) and/or middle cerebral artery (MCA) might be occluded. It is not reasonable to select ACAs and/or MCAs as the reference artery automatically.

Radiation exposure is unavoidable in perfusion CT. The radiation dose is increased because of repeated scans. Radiation dose can be reduced by dose-reduction method which allows the effective dose to be reduced half comparable to the total dose for conventional perfusion CT in 256 or 640slice whole brain CT perfusion [12]. Drawing the same level and size of ROI before and after $\mathrm{MBH}$ revascularization surgery accurately is rather challenging; therefore, in this study, perfusion changes were measured between each ipsilateral side region and pons control region, and the relative perfusion values before and after the surgery were compared to minimize individual variability. What is more, absolute values of CTP parameters can be calculated by CTP software just like Xe-CT $[13,14]$. However, CBF and CBV vary in a large scale due to individual differences and technical parameters, and can only generate semiquantitative analysis of CT perfusion [15]. The relative perfusion value mentioned above is an effective method of measuring the degree of underperfusion present in a given case or location. Sometimes absolute values of $\mathrm{CT}$ perfusion data may provide deviated information according to the individual difference of degree of internal carotid artery occlusion and sometimes, presence of posterior cerebral artery (PCA) occlusion, and if both sides are of the similar improvement in cerebral perfusion at postoperative evaluation, it may underestimate the improvement of cerebral blood flow. CTP is more accurate for assessing unilateral MMD than bilateral one if the traditional reference region is used. Therefore, we measured perfusion changes between each ipsilateral side region and pons control region, and the relative perfusion values were more accurate for assessment. Measuring the true AIF is rather a tough work in MMD cases. In the CBF calculations with CT perfusion imaging, the choice of a reference artery is of vital importance. AIF is calculated from major cerebral vessels [16]. The anterior circulation artery, such as ACA or MCA, is often selected as the reference artery automatically. However, in MMD patients, ACAs and/or MCAs might be occluded. Therefore, we believe that it is optimal to manually select basilar artery as reference artery, because PCA is seldom affected by revascularization surgery [17]. Finally,
Table 3 Comparison of CT perfusion parameters between preoperative and postoperative 6 months after $\mathrm{MBH}$ indirect revascularization

The data are presented as mean \pm $\mathrm{SD} ; n=57$

$* P<0.05$ (statistically significant)

\begin{tabular}{llll}
\hline & Preoperative operation & Postoperative 6 months & $p$ Value \\
\hline $\mathrm{CBF}\left(\mathrm{ml} \cdot 100 \mathrm{~g}^{-1} \cdot \mathrm{min}^{-1}\right)$ & $54.65 \pm 7.78$ & $69.85 \pm 3.68$ & $0.000^{*}$ \\
$\mathrm{rCBF}$ & $0.68 \pm 0.44$ & $0.78 \pm 0.74$ & $0.009^{*}$ \\
$\mathrm{CBV}\left(\mathrm{ml} \cdot 100 \mathrm{~g}^{-1}\right)$ & $5.68 \pm 2.45$ & $6.12 \pm 2.52$ & 0.056 \\
$\mathrm{rCBV}$ & $0.76 \pm 0.07$ & $0.89 \pm 0.03$ & $0.019^{*}$ \\
MTT(s) & $6.42 \pm 0.91$ & $4.91 \pm 0.82$ & $0.000^{*}$ \\
$\mathrm{rMTT}$ & $1.67 \pm 0.63$ & $1.13 \pm 0.25$ & $0.008^{*}$ \\
TTP(s) & $20.62 \pm 0.91$ & $15.46 \pm 0.56$ & $0.000^{*}$ \\
$\mathrm{rTTP}$ & $1.82 \pm 0.24$ & $1.30 \pm 0.13$ & $0.012^{*}$ \\
\hline
\end{tabular}


64-slice multi-detector CT scanner has a limited spatial coverage. So, in our study, we used volume shuttle scanning mode to display range from basal ganglia region to brainstem region, which can get $80 \mathrm{~mm}$ cerebral perfusion image in one time. However, the 256- or 640-slice CT [18], which enables the entire brain to be scanned in a single examination with administration of one contrast medium bolus, does not have the spatial coverage limitations and can be used to obtain the whole brain perfusion data.

For the diagnosis of hemispheric infarcts and hemorrhage, brain CT scanning is the most common and primary imaging technique. Compared with MR angiography, CT angiography could be easily performed for MMD patients at much lower costs, and without the necessity to transfer the patient to another imaging unit. Compared with PET and SPECT, CTP is more readily accessible for the evaluation of cerebral perfusion [14]. CTP is characterized by nonradioactive nuclides, high spatial resolution, short examination time, low cost, and low technology requirements. Compared with other methods, perfusion CT can detect the size of hypoperfused regions rapidly when there is an acute stroke and a lack of significant contraindications. What is more, CTP examination is of a short duration, making it an ideal technique for postsurgical patients without artifacts of movement, susceptibility, and hemorrhage. Meanwhile, CTA images are reconstructed from source data that are acquired with dynamic scans. Both CTA images and quantitative method of measuring cerebral hemodynamics are performed in one examination. CTA can be performed to examine the morphologic status of cerebral arteries. Although deemed as the "gold standard" imaging modality for the diagnosis and postoperative follow-up of MMD, the invasive nature of cerebral angiography means a number of limitations, including procedural risks, costs, and limited availability. As a result, it is less desirable than CTA for screening of intracranial stenosis and occlusion or following patients with these conditions.

In this study, to our surprise, we noted that the ischemic symptoms of some MMD patients improved at very early time (15-20 days) post operation, such as recovered muscle strength and decreased frequency of TIA, etc. Generally speaking, the major disadvantage of indirect revascularization was delayed collateral blood circulation after treatment. Calamante et al. [16] believed that the beneficial effects are not immediate because surgical collaterals require 3-4 months to develop and there is a potential risk of perioperative ischemic stroke. However, based on this study's results, significant statistical differences were observed in the TTP and rTTP at an early time (21 days) before and after $\mathrm{MBH}$ indirect revascularization on ischemic MMD patients. TTP referred to the length of time for brain tissue to reach peak density enhancement. Compared to the brain blood flow before $\mathrm{MBH}$, new collaterals from ECA system to cortical region in hole areas of ischemic symptomatic hemispheres came out after the operation. So, the length of time for brain tissue to reach peak density enhancement was sensitive. Yun et al. [12] also declared that changes in TTP values can be used to predict clinical outcomes after revascularization surgery. Theoretically, the blood supply for the brain tissues outside the middle cerebral arterial perfusion region in this study was obtained from $\mathrm{MBH}$ indirect revascularization, which is relatively easy and safe, and can create transgaleal and/or transdural anastomoses from external arteries to a hypoperfused brain via angiogenesis. This surgery is widely acknowledged to be effective in the treatment of MMD in humans. Ohmori et al. [19] used a rat model, which was more similar to human chronic hypoperfused cerebrovascular diseases (such as MMD), to evaluate the angiogenetic effect of indirect bypass surgery. The results demonstrated that the $\mathrm{CBF}$ and number of small vessels increased significantly 21 days after bilateral internal carotid artery occlusion. Immunohistochemical findings of tissues collected 21 days post-BICAO in animals that received indirect bypass showed a blurred boundary between the cerebral cortex, arachnoid, fibrous coat, and galeal tissue. Numerous vessels smaller than $50 \mu \mathrm{m}$ were found at the boundary zone, giving the appearance of a thin fibrous coat and extending to the cortex and white matter. So we speculated that the early change of symptoms might be related with capillary anastomosis in ischemic cortical region after indirect revascularization, which reduced the Moyamoya vascular load of compensatory hyperplasia. At the early time (15-20 days), the subtle change cannot be monitored by the conventional CT, MRI, and even DSA; however, CTP, as a smart method to obtain functional information about cerebral blood flow, can sensitively detect subtle abnormal hemodynamics. TTP maps have the capability of being quite sensitive to the presence of altered brain perfusion, thus TTP and rTTP values can catch the very subtle change at very early time, and rTTP seems to be more accurate for assessment than absolute TTP value.

\section{Conclusions}

In summary, CTP is a sensitive method to obtain functional imaging of cerebral microcirculation, which can be a noninvasive assessment of the abnormalities of intracranial arteries and cerebral perfusion changes in MMD before and after surgery. Normalized data (relative values) were more sensitive and accurate than absolute values of $\mathrm{CT}$ perfusion data. TTP and CBF map, especially the relative values, seem to have the capability of being quite sensitive to the presence of altered brain perfusion at early time after indirect revascularization. From CTP, we confirmed MBH surgery as a useful method of indirect revascularization, which can be applied as a simple and effective method for the improvement 
of symptoms and prevention of stroke events in ischemic MMD.

Acknowledgments This work was supported by the National Natural Science Foundation of China (project no. 8127127130973102 and 81171093 ) and "1255" Discipline Construction Project of Changhai Hospital (CH125520105).

Conflict of interest We declare that we have no conflict of interest.

Open Access This article is distributed under the terms of the Creative Commons Attribution License which permits any use, distribution, and reproduction in any medium, provided the original author(s) and the source are credited.

\section{References}

1. Baba T, Houkin K, Kuroda S (2008) Novel epidemiological features of moyamoya disease. J Neurol Neurosurg Psychiatry 79:900-904

2. Fukui M (1997) Guidelines for the diagnosis and treatment of spontaneous occlusion of the circle of Willis ('moyamoya' disease). Research committee on spontaneous occlusion of the circle of Willis (Moyamoya Disease) of the ministry of health and welfare, Japan. Clin Neurol Neurosurg 99(suppl 2):S238-S240

3. Han DH, Kwon OK, Byun BJ, Choi BY, Choi CW, Choi JU, Choi SG, Doh JO, Han JW, Jung S, Kang SD, Kim DJ, Kim HI, Kim HD, Kim MC, Kim SC, Kim SC, Kim Y, Kwun BD, Lee BG, Lim YJ, Moon JG, Park HS, Shin MS, Song JH, Suk JS, Yim MB (2000) A co-operative study: clinical characteristics of 334 Korean patients with moyamoya disease treated at neurosurgical institutes. Acta Neurochir (Wien) 142:1263-1273

4. Scott RM, Smith ER (2009) Moyamoya disease and moyamoya syndrome. N Engl J Med 360:1226-1237

5. Rim NJ, Kim HS, Shin YS, Kim SY (2008) Which CT perfusion parameter best reflects cerebrovascular reserve?: correlation of acetazolamide-challenged CT perfusion with single-photon emission CT in moyamoya patients. AJNR 29:1658-1663

6. Scott RM, Smith JL, Robertson RL, Madsen JR, Soriano SG, Rockoff MA (2004) Long-term outcome in children with moyamoya syndrome after cranial revascularization by pial synangiosis. J Neurosurg 100(2 Supp):142-149

7. Togao O, Mihara F, Yoshiura T, Tanaka A, Noguchi T, Kuwabara Y, Kaneko K, Matsushima T, Honda H (2006) Cerebral hemodynamics in moyamoya disease: correlation between perfusion-weighted MR imaging and cerebral angiography. AJNR 27:391-397
8. Touho H, Karasawa J, Ohnishi H (1996) Preoperative and postoperative evaluation of cerebral perfusion and vasodilatory capacity with 99 mTc-HMPAO SPECT and acetazolamide in childhood moyamoya disease. Stroke 27:282-289

9. Wintermark M, Thiran JP, Maeder P, Schnyder P, Meuli R (2001) Simultaneous measurement of regional cerebral blood flow by perfusion CT and stable xenon CT: a validation study. AJNR 22: 905-914

10. Miles KA, Hayball M, Dixon AK (1991) Colour perfusion imaging: a new application of computed tomography. Lancet 337:643-645

11. Wintermark M, Sesay M, Barbier E, Borbély K, Dillon WP, Eastwood JD, Glenn TC, Grandin CB, Pedraza S, Soustiel JF, Nariai T, Zaharchuk G, Caillé JM, Dousset V, Yonas H (2005) Comparative overview of brain perfusion imaging techniques. Stroke 36:e83-e99

12. Yun TJ, Cheon JE, Na DG, Kim WS, Kim IO, Chang KH, Yeon KM, Song IC, Wang KC (2009) Childhood moyamoya disease: quantitative evaluation of perfusion MR imaging correlation with clinical outcome after revascularization surgery. Radiology 251(1): 216-223

13. Eastwood JD, Lev MH, Lee TY, Barboriak DP, Delong DM, Fitzek C, Herzau M, Wintermark M, Meuli R, Brazier D, Provenzale JM (2002) CT perfusion scanning with deconvolution analysis: pilot study in patients with acute middle cerebral artery stroke. Radiology 222:227-236

14. Eastwood JD, Lev MH, Wintermark M, Fitzek C, Barboriak DP, Delong DM, Lee TY, Azhari T, Herzau M, Chilukuri VR, Provenzale JM (2003) Correlation of early dynamic CT perfusion imaging with whole-brain MR diffusion and perfusion imaging in acute hemispheric stroke. AJNR 24:1869-1875

15. Leenders KL, Perani D, Lammertsma AA, Heather JD, Buckingham P, Healy MJ, Gibbs JM, Wise RJ, Hatazawa J, Herold S (1990) Cerebral blood flow, blood volume and oxygen utilization: norm al values and effect of age. Brain 113:27-47

16. Calamante F, Ganesan V, Kirkham FJ (2001) MR perfusion imaging in moyamoya syndrome: potential implications for clinical evaluation of occlusive cerebrovascular disease. Stroke 32: 2810-2816

17. Calamante F, Thomas DL, Pell GS, Wiersma J, Turner R (1999) Measuring cerebral blood flow using magnetic resonance techniques. J Cereb Blood Flow Metab 19:701-735

18. Zhang J, Wang J, Geng D, Li Y, Song D, Gu Y (2013) Whole-brain $\mathrm{CT}$ perfusion and $\mathrm{CT}$ angiography assessment of moyamoya disease before and after surgical revascularization: preliminary study with 256-slice CT. PLoS One 8(2):e57595

19. Ohmori Y, Morioka M, Kaku Y, Kawano T, Kuratsu J (2011) Granulocyte colony-stimulating factor enhances the angiogenetic effect of indirect bypass surgery for chronic cerebral hypoperfusion in a rat model. Neurosurgery 68(5):1372-1379 